\title{
COMPETENCIA DISCURSIVA Y REDACCIÓN DE ABSTRACTS: GESTIONAR LA DOBLE IDENTIDAD ESTUDIANTE E INVESTIGADOR ${ }^{1}$
}

\author{
DISCOURSE COMPETENCE AND ABSTRACT WRITING: \\ MANAGING THE DUAL IDENTITY OF STUDENT AND \\ RESEARCHER
}

\author{
MARIA DOLORS CAÑADA PUJOLS \\ Universitat Pompeu Fabra \\ mariadolors.canada@upf.edu
}

CARME BACH MARTORELL

Profesora agregada Serra Húnter

Universitat Pompeu Fabra

Carme.bach@upf.edu

\section{RESUMEN}

Los abstracts de trabajos de fin de grado son textos académicos redactados por estudiantes que se inician en la investigación. Aunque existen estudios sobre los abstracts producidos por expertos, son escasos los dedicados a producciones de estudiantes. En este artículo se analizan los abstracts redactados por futuros egresados en Lenguas Aplicadas en tres niveles de análisis: supratextual, macrotextual (movimientos retóricos) y microtextual (marcadores metadiscursivos). El análisis muestra la aparición de estructuras retóricas prototípicas del género, al igual que algunos movimientos emergentes. En cuanto a los recursos metadiscursivos, los estudiantes usan una gran variedad de marcadores, en la que destacan los metadiscursivos estructuradores (encapsuladores y ordenadores discursivos).

${ }^{1}$ Este trabajo forma parte del proyecto de investigación ECODAL: Evaluación de la competencia discursiva de aprendices adultos plurilingües: detección de necesidades formativas y pautas para un aprendizaje autónomo ( ${ }^{\circ}$ de ref. EDU2016-75874-P), del Plan Nacional de Investigación Científica, Desarrollo e Innovación Tecnológica 2016 del Ministerio de Economía, Industria y Competitividad (MINECO), cofinanciado por la Agencia Estatal de Investigación (AEI) y el Fondo Europeo de Desarrollo Regional (FEDER). Las autoras forman parte de Gr@el, grupo de investigación consolidado y financiado por la Generalitat de Catalunya (AGAUR, 2017SGR 915). Agradecemos muy sinceramente la labor de critical friends llevada a cabo por Andreína Adelstein e Iria da Cunha. 
La conclusión principal es que los abstracts analizados son textos híbridos, a medio camino entre el discurso académico, producido por un estudiante que va a ser evaluado, y un discurso especializado, producido por y dirigido a lectores expertos.

Palabras clave: Escritura académica, abstracts, movimientos retóricos, marcadores metadiscursivos.

\begin{abstract}
Undergraduate final-year dissertations abstracts are academic texts written by students starting out as researchers. Although there are studies on abstracts produced by experts, few of them have dealt with abstracts produced by students. In this paper, abstracts written by future graduates in Applied Languages are analysed. This is done at three levels: supratextual, macro-textual (rhetorical moves) and micro-textual (metadiscourse markers). The analysis shows how rhetorical structures that are prototypical of the genre appear, as do some emergent moves. Regarding metadiscourse resources, students use a wide range of markers, the most prominent ones being metadiscourse structure markers (encapsulators —also known as shell nouns — and ordinal markers). The main finding is that the abstracts are hybrid texts: halfway between academic discourse, produced by a student to be assessed, and specialised discourse, produced by and directed to expert readers.
\end{abstract}

Keywords: Academic writing, abstracts, moves, metadiscourse markers.

Recibido: 13/10/19. Aceptado: 27/03/2020.

\title{
1. INTRODUCCIÓN
}

T os resúmenes o abstracts $^{2}$ son discursos académicos especializados que se relacionan con la elaboración y la transmisión del saber (al igual que la ponencia, la síntesis o el artículo de investigación). La literatura científica ofrece numerosos estudios sobre el género académico abstract a partir de corpus de textos redactados por investigadores expertos de diversas disciplinas (Cavalieri, 2014; Dos Santos, 1996; Lorés y Bondi, 2014; Saeeaw y Tangkiengsirin, 2014), que, obviamente, dominan el género. Sin embargo, existen pocos trabajos de investigación que analicen producciones de estudiantes (Aktas y Cortes, 2008; Díez Prados, 2018) y que permitan evaluar su grado de dominio del género, a pesar de que Graetz (1985: 125) ya señalaba que "Abstracts continue to remain a neglected field

${ }^{2}$ De ahora en adelante utilizaremos el término inglés por ser el más utilizado en la literatura científica. 
among discourse analysts. This is unfortunate as they are texts particularly suited to genre investigation. Moreover, study of certain types of abstracts can potentially be highly revealing of disciplinary discourse communities, particularly when the abstracts comprise the evidence on which gate-keeping decisions are made".

En este artículo se analizan los abstracts producidos por futuros egresados en Lenguas Aplicadas, que incluyen en sus trabajos de fin de grado. La importancia de estudiar estos productos textuales viene dada por el hecho de que según el Real Decreto 1393/2007, de 29 de octubre, por el que se establece la ordenación de las enseñanzas universitarias oficiales, todos los nuevos planes de estudios deben incluir la programación de un proyecto final de Grado o de Máster. Es decir, que la exigencia de un trabajo final no es propia de las universidades o de las titulaciones españolas, sino que es un requisito estatal para todos los candidatos a egresados. Este contexto provoca la emergencia de un género nuevo con una presencia importante en las prácticas académicas escritas que merece una investigación descriptiva como la que se aborda en este artículo. Las implicaciones para la didáctica de la escritura académica son evidentes.

\section{1. ¿Qué es un abstract?}

El abstract es un tipo de resumen que encabeza los artículos científicos. Habitualmente se incluye dentro del mismo artículo, después del título y en la primera página. Su función es informar sobre el contenido del texto, para que los posibles lectores puedan hacerse en poco tiempo una idea general de su contenido y decidir si les interesa leer el documento entero. Formalmente, es un resumen básicamente informativo que sintetiza las aportaciones más destacadas del artículo: el tema que se propone estudiar, la metodología aplicada, y, sobre todo, los resultados obtenidos (Cassany y López-Ferrero, 2000).

El abstract pertenece a la familia de géneros académicos, es decir, "los que se producen en universidades, institutos de investigación científica y academias, que refieren a temáticas propias de un dominio científico y que responden a convenciones y tradiciones retóricas específicas de cada área de conocimiento" (Adelstein y Kuguel, 2005). La familia de géneros académicos incluye textos tanto orales (conferencia, presentación oral, etc.) como escritos (ensayo, comentario personal, revisión bibliográfica, entre otros) y se caracteriza por una gran variación disciplinar (Venegas, Zamora y Galdames 2016).

El trabajo de fin de grado (TFG de ahora en adelante) ${ }^{3}$ es un género académico de carácter evaluativo (Venegas et al., 2016), ya que recibirá una determinada

${ }^{3}$ La denominación oficial adoptada en España es la de Trabajo Final de Grado (TFG) y es la que utilizaremos en este artículo; en otros contextos también se usan distintas denominaciones: 'Tesis de grado', Undergraduate dissertation, Undergraduate Thesis, Undergraduate Research Project, Undergraduate Final Year Project, o Final Year Research Project. 
calificación por parte de los docentes, algo que no ocurre con los textos científicos; el abstract comparte con el TFG ese mismo carácter. Según estos autores, existen tres tipos de abstracts: a) descriptivos, b) estructurados y c) informativos. Los abstracts descriptivos refieren, de modo muy breve (alrededor de 100 palabras), el trabajo que se ha realizado; son más frecuentes en programas de congresos que en revistas científicas. Los abstracts estructurados se caracterizan por presentarse como una lista de enunciados correspondientes a los siguientes apartados: objetivos, diseño, lugar y circunstancias, pacientes (u objetivo del estudio), intervención, mediciones y principales resultados y conclusiones. Por último, los abstracts informativos son los más frecuentes en los contextos científicos (revistas, actas de congresos, por ejemplo) y académicos (TFG, trabajos de fin de máster, tesis doctorales, entre otros). Su extensión suele situarse entre 150 y 200 palabras y actúan como "sustitutos" de los documentos completos.

Tanto la organización retórica de los abstracts como sus otras características discursivas no pueden entenderse sin describir las funciones que se asocian al género. En primer lugar, permiten la indexación del texto en distintas bases de datos, tanto a nivel local (repositorios electrónicos de las universidades, por ejemplo) como internacional (por ejemplo, Linguistics and Language Behavior Abstracts o Web of Science). En segundo lugar, orientan al lector en cuanto al contenido básico del texto completo: objetivos, metodología, resultados, etc. Por último, tienen una clara función persuasiva, ya que uno de los objetivos que persiguen es convencer al lector para que aborde la lectura completa del texto. En palabras de Jiang y Hyland (2018: 1): "Research article abstracts have become an important genre in all knowledge fields, playing a crucial role in persuading readers, and reviewers, to take the time to go further into the paper itself. This promotional aspect of abstracts is well known (...)."

\subsection{Estructura retórica de los abstracts}

La estructura tanto de los artículos como de los abstracts de textos académico-científicos ha sido abundantemente tratada en la literatura. En este artículo, siguiendo los trabajos de Swales (1990) y Bathia (1993), entendemos por movimiento retórico o movida cada fragmento particular de texto con un objetivo comunicativo concreto ${ }^{4}$. Así pues, como se ha dicho, un abstract debe seguir la macroestructura del texto original, de manera que la estructura y los contenidos de ambos textos han de ser muy similares: “(...) este [el trabajo académico] incluye unos contenidos prototípicos en un orden determinado, como motivación, objetivos, estado de la cuestión, marco teórico, metodología, análisis, resultados y conclusión. Así, el resumen de este gé-

\footnotetext{
${ }^{4}$ Aunque Parodi (2010 a y b) propone también el término macromovida para referirse a agrupaciones de movidas que cumplen una misma función retórica en el texto, no parece pertinente recurrir a él para el análisis del corpus, dado que son textos muy breves en los que no se observa agrupación de movimientos.
} 
nero textual debe reflejar también los contenidos principales del trabajo, de manera muy breve (...) y en el mismo orden" Da Cunha (2016: 108).

En la misma línea, Sancho (2014) indica que todo abstract debe incluir: introducción (I), metodología (M), resultados (R) y discusión (D). Este autor considera asimismo que el abstract debe seguir la estructura típica de muchos artículos de investigación, ya señalada por Swales (1990): la estructura IMRD, acrónimo correspondiente a los movimientos retóricos identificados en los textos: Introduction, Methodology, Results, Discussion y Conclusion, parecida a la estructura indicada para dichos artículos por Bathia (1993), quien en el primer movimiento incluye los objetivos de la investigación.

Dos Santos (1996), en una investigación sobre abstracts de artículos especializados en el ámbito de la lingüística aplicada, indica la presencia de un quinto movimiento, el primero desde el punto de vista secuencial y no descrito con anterioridad, que permite situar la investigación, refiriéndose al estado actual del conocimiento, citando investigaciones previas o planteando un determinado problema. En este artículo se sigue la propuesta de Dos Santos (1996), que además de incluir las propuestas anteriores se sitúa en el mismo ámbito de conocimiento, el ámbito de las lenguas aplicadas.

Cabe señalar que Venegas, Núñez, Zamora y Santana (2015) introdujeron el término movidas emergentes para referirse a movimientos no descritos en los estudios sobre un determinado género. En este artículo se usa el término movimientos emergentes para hacer referencia a aquellos movimientos retóricos no descritos en el apartado anterior que se han identificado en el corpus de análisis.

\subsection{Elementos metadiscursivos}

Los elementos metadiscursivos son definidos por Hyland (2005) como una categoría que pretende analizar los textos desde el punto de vista de la interacción del autor con su texto (función textual interactiva) y con sus lectores (función interpersonal interaccional). En este artículo se analizarán únicamente los elementos metadiscursivos con función textual presentes en el corpus; es decir, aquellos destinados a la construcción de un texto coherente para el lector: "The use of resources in this category therefore addresses ways of organizing discourse, rather than experience, and reveals the extent to which the text is constructed with the reader's needs in mind" (Hyland, 2005: 49), ya que su presencia en el corpus es considerable y merecen, por lo tanto, un estudio pormenorizado.

Según Hyland (2005) existen cinco tipos de recursos metadiscursivos textuales: transition markers (marcadores de transición, en adelante), endophoric markers (marcadores endofóricos), evidentials (evidenciadores), code glosses (marcadores de contextos definitorios) y frame markers (denominados marcadores estructurales en este artículo). 
Se incluyen en el grupo de los marcadores discursivos estructuradores los metadiscursive names que Jiang y Hyland (2018: 509) consideran un rasgo olvidado en los elementos del metadiscurso: "We set out here to correct this oversight and attempt to map what we call metadiscursive nouns onto Hyland's (2005) metadiscourse model, showing how these nouns promote interactions between writers and readers in academic writing". En efecto, estos nombres contribuyen de modo notable en el establecimiento de la cohesión de los textos, ya que permiten la recuperación (tanto anafórica como catafórica) de fragmentos discursivos. Además, como señalan Jiang y Hyland (2018: 520), juegan un papel importante en la construcción de la coherencia textual: "In the interactive dimension, metadiscourse nouns also help to create logical coherence form discoursal cohesion, shaping texts to what readers will find most familiar, plausible, and persuasive".

Estos nombres metadiscursivos ya fueron tratados anteriormente por Vendler (1968), Halliday y Hasan (1976) que los denominaron nombres generales o por Francis (1986), que se refirió a ellos como nombres anafóricos. Más adelante, Schmid (2000 y 2018) los ha llamado shell nouns, mientras que Muñoz y Ciapuscio (2019) proponen para el español el término nombres rotuladores 5 . En el artículo se usará el término nombres encapsuladores que retoma el de shell nouns y que es el más difundido en la bibliografía (Borreguero, 2018; López Samaniego, 2018, entre otros). En el análisis de los datos se profundizará en estos recursos metadiscursivos textuales, mostrando ejemplos del análisis del corpus.

\section{METODOLOGÍA}

El corpus estudiado está compuesto por 36 abstracts incluidos en los TFG de una promoción completa del grado de Lenguas Aplicadas de una universidad catalana. Los textos se recogieron al final del curso académico 2017-2018. Cada uno de los 36 estudiantes redactó tres abstracts: dos en las lenguas propias (catalán y español) y uno en lengua adicional (inglés, mayoritariamente). En este artículo se analizan únicamente los abstracts escritos en español; el corpus consta, pues, de 36 textos, con una extensión de 7.488 palabras.

Los sujetos recibieron instrucciones generales sobre la realización del TFG, pero sin focalizar en la redacción del trabajo en general ni del abstract en particular. La extensión de los abstracts es muy diversa y va desde un mínimo de 83 palabras a un máximo de 327 palabras; la extensión media de los textos es de 208 palabras.

${ }^{5}$ Algunos estudios recientes como el de Parodi y Burdiles (2016) tratan otros mecanismos encapsuladores como los pronombres, que no hemos abordado en este artículo, por considerarlos más cercanos a las anáforas textuales que a los marcadores de estructuración del discurso. 
Para el análisis de las características macrotextuales y microtextuales de los abstracts se ha utilizado la herramienta de análisis QDA (Qualitative Data Analysis) Atlas-ti, que permite un análisis cualitativo mediante el etiquetado de los textos en función de las categorías de análisis que emergen y también un estudio de las frecuencias de los fenómenos objeto de estudio.

Respecto del análisis de los datos, se procedió de la siguiente manera. En primer lugar, se analizó la superestructura textual de los abstracts, es decir los aspectos formales que caracterizan las producciones estudiadas: presencia o ausencia de título(s) y de palabras clave y su naturaleza. Como señalan Martín Peris, Atienza, González, López Ferrero y Torner (2008), las superestructuras organizan cognitivamente el proceso de lectura, comprensión y (re-)producción de los géneros discursivos y son, por lo tanto, un elemento necesario para el desarrollo de la competencia discursiva.

En segundo lugar, se etiquetaron los movimientos presentes en cada abstract, considerando las categorías propuestas por la literatura y también creando nuevas etiquetas cuando en el análisis se observaron movimientos no previstos inicialmente (que se han considerado movimientos emergentes).

En tercer lugar, se etiquetaron las distintas categorías metadiscursivas, siguiendo la propuesta de Hyland (2005) e integrando la categoría de los nombres encapsuladores, propuesta por Jiang y Hyland (2017) y Jiang y Hyland (2018).

Por último, se procedió a relacionar las categorías de ambas dimensiones para llegar a los resultados que se expondrán en el siguiente apartado. Para ello, las dos autoras analizaron la totalidad del corpus y los pocos casos en los que existían discrepancias en cuanto al etiquetado se discutieron hasta alcanzar un acuerdo. Este proceso permite garantizar la fiabilidad de las categorías.

\section{ANÁLISIS DE LOS DATOS}

\subsection{Caracterización de la superestructura textual}

Las investigaciones publicadas hasta la fecha no se refieren a las características formales de los abstracts realizados por estudiantes. Los problemas detectados en este aspecto en el corpus requieren abordar la superestructura de los textos. En cualquier publicación científica, el texto del abstract propiamente dicho se presenta precedido por el título abstract, resumen, résumé, según la lengua de redacción y casi siempre seguido por una serie limitada de keywords, palabras clave o mots clé. Se trata, pues, de un esquema preestablecido convencionalmente que comparten los usuarios que dominan el género.

Del análisis se desprende que casi todos los estudiantes (83,3\%) utilizan en sus TFG el término resumen para encabezar el texto. Se observa que tres de los estudiantes en lugar del título indican la lengua de redacción del abstract (español), con lo 
que se posicionan claramente como estudiantes que siguen las instrucciones recibidas ${ }^{6}$ : en la guía docente de la asignatura Trabajo de fin de grado se indica que este "deberá incluir un resumen de unas 250 palabras en tres lenguas: español, catalán e inglés”. Se trata de una clara manifestación de la identidad estudiante frente a la identidad investigador, que se puede interpretar como un problema de identificación de los parámetros situacionales del género que se les pide que escriban.

Por otra parte, casi un $64 \%$ de los textos van seguidos de palabras clave, mientras que el restante $36 \%$ no las incluye. El uso de palabras clave es particularmente importante para la búsqueda y consulta de los documentos y tiene, por lo tanto, una clara función de promoción. Sin embargo, cabe señalar que el concepto no ha sido comprendido por parte de todos los autores que, en ocasiones, utilizan términos que en su opinión caracterizan su TFG pero que no pertenecen a la disciplina en la que se sitúan sus investigaciones; por poner solo algunos ejemplos: creación, organismos, alto valyrio, Mallorca.

En definitiva, del análisis se desprenden problemas de dominio del género, que la enseñanza de estos textos académicos debe tomar en cuenta.

\subsection{Caracterización macrotextual}

El análisis macrotextual de los resúmenes arroja un total de siete movimientos: tema, objetivos, metodología, resultados, conclusiones, estructura del trabajo y limitaciones. Corresponden al prototipo de estructura retórica establecido por Dos Santos (1996) los cinco primeros, mientras que los movimientos estructura y limitaciones son movimientos emergentes, según el término acuñado por Venegas et al. (2015). A pesar de la importancia que tiene el lugar que ocupa el movimiento en el texto (cfr. Da Cunha 2016), en esta investigación no se abordará este parámetro.

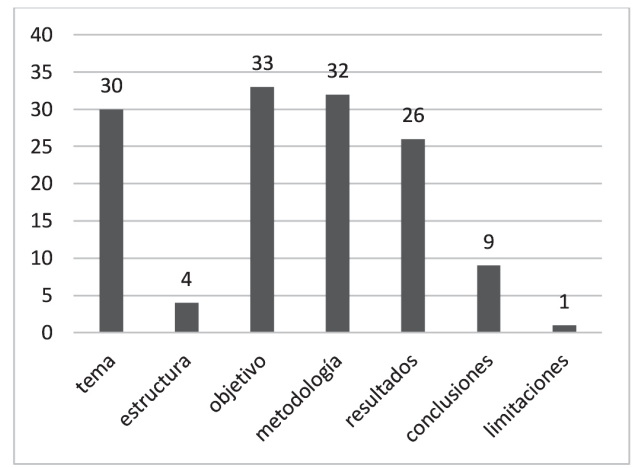

Gráfico 1. Frecuencia de aparición de los movimientos retóricos.

\footnotetext{
${ }^{6}$ Un estudiante incluye el título Resum en catalán y otros dos no incluyen título alguno.
} 
Como se puede observar en el Gráfico 1, los movimientos más frecuentes son la presentación de los objetivos, de la metodología y del tema. Cabe precisar que el número de movimientos de cada uno de los textos es variable: el $75 \%$ de los textos del corpus tiene entre 4 y 5 movimientos, aunque otros incluyen solo uno (objetivos o tema), y otros solo 2 ó 3. Además, diversos estudiantes "desdoblan" los movimientos en sus abstracts, por ejemplo, mencionando en dos lugares distintos el tema (4 estudiantes), los objetivos (5 estudiantes) o la metodología (5 estudiantes), lo que pone de manifiesto una organización reiterada poco propia de textos muy sintéticos. A continuación, se analizan en detalle los movimientos identificados.

En primer lugar, el tema - situating the research según Dos Santos (1996)- es uno de los movimientos retóricos más frecuentes (30/36 ocurrencias en el corpus) y ocupa una extensión variable (entre 23 y 149 palabras, con una media de 73.3 palabras) y considerable (en un tercio de los textos, el tema supone entre el $40 \mathrm{y}$ el 60\% del total de palabras). El objetivo principal de este movimiento es atraer la atención del lector "to sell the research being offered" (Dos Santos, 1996: 485). Este autor identificó en su corpus cuatro posibles submovimientos: a) indicar el estado actual del conocimiento, b) citar investigaciones previas, c) ampliar investigaciones previas, y d) exponer un problema, siendo el primero y el último los más frecuentes según su estudio.

El tema aparece en los abstracts como el primero de los movimientos. Por lo general, los estudiantes empiezan por plantear el tema e introducir a continuación la motivación o justificación de su TFG, es decir, exponen el vacío de conocimiento que su trabajo se propone llenar. En el ejemplo (1), la estudiante plantea el tema (los programas de mensajería instantánea como medio de comunicación) y expone la necesidad de ampliar la investigación lingüística, en concreto relacionando el género escrito con el registro oral coloquial, que constituirá el objetivo de su TFG:

(1) En poco más de quince años, los programas de mensajería instantánea se han convertido en el medio de comunicación escrita más popular alrededor del mundo. Sin embargo, no ha sido hasta hace poco que estudiosos de la lengua han comenzado a interesarse por las características lingüísticas de este género, y aún son pocos los estudios que lo han relacionado con el lenguaje coloquial oral. ES_12

Del total de los 36 abstracts, 15 incluyen el submovimiento justificación 7 para destacar la pertinencia del estudio realizado, a menudo introducido por marcadores de oposición o causa, como en los dos ejemplos siguientes:

${ }^{7}$ Se opta por situar esta justificación dentro del movimiento tema porque en la mayoría de los casos no se trata de frases completas sino únicamente proposiciones, como en los ejemplos (4 y 5). 
(2) pero su efecto desde el punto de vista de las diferencias individuales no se ha identificado. ES_02

(3) ya que actualmente es un campo que cuenta con poca literatura. ES_22

La exposición del tema manifiesta en muchos casos la identidad del autor más como estudiante que como investigador, puesto que no sitúan de modo breve el ámbito de conocimiento en el que se enmarca el trabajo, sino que muestran extensamente su saber, lo que suponen meritorio para una evaluación positiva por parte del docente. En el ejemplo (4), la autora sitúa su trabajo en el ámbito del aprendizaje del léxico en lengua extranjera, pero aborda una exposición histórica excesivamente extensa ( $40 \%$ del total) para un texto caracterizado por su brevedad:

(4) Se ha demostrado que el vocabulario es uno de los aspectos más complicados y también más cruciales en el aprendizaje de una lengua extranjera. A pesar de su importancia, los expertos no han empezado a investigar este ámbito hasta las últimas décadas. Los primeros estudios trataban el vocabulario relacionado con aspectos como los estilos o las estrategias de aprendizaje o características personales. Recientemente, algunos psicolingüistas han introducido elementos como la memoria o la dominancia cerebral en el aprendizaje de vocabulario en la L2, pero su efecto desde el punto de vista de las diferencias individuales no se ha identificado. ES_02

En segundo lugar, casi todos los estudiantes se refieren a los objetivos del TFG (33 ocurrencias, 91,6\%), movimiento retórico que también incluyen los expertos. Se trata, por lo general, de un movimiento poco extenso que suele limitarse a una o dos frases, como en el ejemplo siguiente:

(5) con este trabajo se pretende dar un paso más en el estudio de la lengua de signos catalana (LSC) en cuanto a la adquisición de esta, concretamente en la adquisición de la negación. ES_06

La exposición de la metodología también aparece en la mayoría de los abstracts $(88,8 \%)$, solo 4 de los estudiantes no la incluyen. En este movimiento se mencionan distintos elementos: el paradigma de investigación (una investigación cualitativa), los instrumentos de recogida de datos (entrevistas en profundidad, observaciones no participantes, cuestionarios sociolingüisticos, encuestas, historias de vida), la extensión del corpus (El corpus de análisis contiene 813 neologismos formales, semánticos y préstamos) y las variables consideradas. A menudo, esta información sobre la metodología adoptada peca de un grado de especificación excesivo, poco adecuado para el carácter sintético del género. En el ejemplo (6) la especificación de las variables es innecesaria, del mismo modo que en el ejemplo (7) también lo son los detalles que se dan sobre el diseño del cuestionario: 
(6) Los factores analizados son el tiempo vivido en Barcelona, el lugar de origen de los interlocutores más habituales, el grado de formalidad de las situaciones y la ideología de los hablantes. ES_27

(7) Dicho cuestionario está compuesto de un total de 26 preguntas (11 cerradas y 15 abiertas); se estructuró en 3 grandes bloques: procedencia, motivación y usos y estilos de aprendizaje. ES_30

Contrariamente a lo observado por Dos Santos (1996), los estudiantes se refieren a menudo a los informantes, también con un elevado grado de detalle, como en los dos ejemplos siguientes:

(8) los informantes (dos grupos de chicas de origen marroquí: diez nacidas en Cataluña, y diecisiete que llegaron a la comunidad autónoma en la infancia o preadolescencia). ES_05

(9) se ha entrevistado a seis participantes - cinco alumnos y una docentede la clase de 2. ${ }^{\circ} \mathrm{B}$ de alemán de la Escola Oficial d'Idiomes (EOI) Cornellà de Llobregat. ES_14

En cuanto al movimiento resultados, cabe decir que no es tan frecuente como los anteriores (26/36 textos) y que su finalidad es resumir brevemente los principales hallazgos de la investigación. Un ejemplo típico de la exposición de resultados se da en (10).

(10) Los resultados del estudio demuestran que, en efecto, sí que es posible reconocer correctamente una voz desconocida y que, además, las mujeres, de manera significativa, reconocen correctamente una voz de hombre con más frecuencia que una voz de una persona de su mismo sexo, hecho que se podría explicar porque nuestro nivel de atención hacia una voz es más alto cuando la voz pertenece a una persona del sexo opuesto. ES_31

En la mitad de los casos (47,2\%), el movimiento se introduce mediante la formulación "Los resultados muestran / demuestran levidencian" o con cambios en el sujeto como en "Los datos también muestran que" o "el análisis cuantitativo demuestra", con un claro procedimiento de tematización. Solo en tres ocasiones se emplean enunciados en primera persona (hemos comprobado) o en forma impersonal (se ha podido observar que).

Por último, las conclusiones son poco frecuentes (9/36, que corresponde al $25 \%$ del total), lo que permite suponer un déficit en los futuros egresados en cuanto al dominio de la función cognitiva de razonamiento y también en cuanto al dominio del género. Dicho de otro modo, los estudiantes encuentran dificultades en estructurar los resultados que han conseguido en su investigación para alcanzar 
una información de nivel superior. Las conclusiones aparecen siempre cerrando el abstract y aportan información explicativa para los resultados presentados en el movimiento resultados. No se han observado recurrencias en cuanto a las formas lingüísticas que se emplean para presentar los resultados. A continuación, se incluye un ejemplo extraído del corpus:

(11) Finalmente, las principales conclusiones fueron que no existe una profesión asociada al perfil de estudiante de árabe, mientras que la principal motivación detectada si fue cultural o familiar. Por último, los principales usos del lenguaje coinciden con el estilo de aprendizaje favorito, esto es, la conversación. ES_30

Para concluir el análisis de la estructura retórica de los abstracts, cabe hacer referencia a dos movimientos que la literatura sobre el tema no menciona (movimientos emergentes según Venegas et al., 2015) y que se explican por el hecho de que los autores son estudiantes y no investigadores expertos: la explicitación de la estructura del TFG y sus limitaciones. Se trata de movimientos con un índice de frecuencia bajo $(11,1 \%)$ pero que es preciso describir para poder caracterizar la competencia discursiva de los autores de los textos. El primero de estos movimientos es el denominado estructura del trabajo, del que se presenta a continuación un ejemplo y que, en algún caso, incluso remite a los anexos del trabajo:

(12) Así pues, este trabajo incluye una contextualización sobre la terminología y su marco teórico, una introducción sobre el sector del agua, el proceso de industrialización y la normativa vigente, y un trabajo de corpus. ES_04

La existencia de este movimiento se explica por la confusión entre las características discursivas del abstract y la introducción del trabajo. A pesar de que hay solo cuatro casos de este movimiento en el corpus, podría tratarse de una tendencia más o menos frecuente entre los futuros egresados, ya que, por poner solo un ejemplo, en la sección de ayuda a los estudiantes de la Universidad de Melbourne se recuerda que: "Although it is placed at the beginning of your paper, immediately following the title page, the abstract should be the last thing that you write,

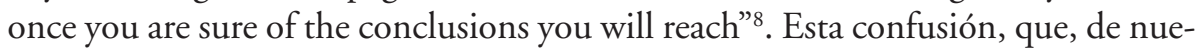
vo, manifiesta el poco dominio de los parámetros situacionales del género, tiene

\footnotetext{
https://services.unimelb.edu.au/_data/assets/pdf_file/0007/471274/Writing_an_Abstract_ Update_051112.pdf
} 
claras incidencias en el nivel textual del abstract. Primero, en el uso de los tiempos verbales, ya que se usa el futuro, un tiempo verbal no adecuado en este género discursivo. Segundo, se usan marcadores estructurales en un tipo de texto que no los requiere (cf. 3.2.2) y que obliga -habida cuenta de su limitada extensión- a reducir la cantidad de información que incluye el abstract. Por consiguiente, esta deficiencia en la estructura retórica puede ser considerada como un problema de interpretación del contexto de enunciación, igual que ocurre con el movimiento limitaciones. En efecto, un único estudiante menciona en su abstract las limitaciones que a su parecer comporta su trabajo, pero, con ello, el autor atenta de algún modo contra la función de promoción típica de este género a la que ya se ha aludido. Se presenta a continuación el fragmento correspondiente a este movimiento:

(13) Aun así, también hay que decir que este estudio no aspira a aportar resultados que se puedan aplicar a toda la realidad lingüística del catalán (...). ES_22

\subsection{Caracterización microtextual}

En este apartado se analizan los elementos metadiscursivos interactivos, es decir, aquellos que guían al lector que se enfrenta a un texto, siguiendo para ello la clasificación de Hyland (2005), que distingue entre marcadores de transición, marcadores estructurales, evidenciadores y marcadores de contextos definitorios. La clasificación de Hyland (2005) incluye asimismo los marcadores endofóricos de los que no se han detectado ocurrencias en el corpus, dada la naturaleza breve del género abstract.

\subsubsection{Marcadores discursivos de transición}

Un marcador de transición (transition marker) es aquel que expresa una determinada relación entre enunciados, lo que en Martín Zorraquino y Portolés (1999) denominan conector y que definen como "aquel marcador discursivo que vincula semántica y pragmáticamente un miembro discursivo con otro miembro anterior"). En la Tabla I se observa la frecuencia de aparición de las 66 ocurrencias identificadas en el corpus, con la presencia de algunos hápax (aunque, consecuentemente, en efecto, especificamente, mientras que, por consiguiente, por el contrario, por ende, recientemente, y) y algunas variantes sintácticas. La mayoría $(68,2 \%)$ de estas formas se encuentra en posición inicial de enunciado y solo un $31,7 \%$ aparecen en posición medial. 
Tabla I. Frecuencia de aparición de los marcadores de transición.

\begin{tabular}{|l|l|l|l|}
\hline a pesar (de) que & 4 & de este modo & 3 \\
\hline actualmente & 2 & en consecuencia & 2 \\
\hline además & 6 & no obstante & 4 \\
\hline asi pues & 2 & Pero & 4 \\
\hline asimismo & 6 & por tanto & 5 \\
\hline aun así & 2 & sin embargo & 5 \\
\hline concretamente & 2 & también & 3 \\
\hline de esta manera & 3 & & \\
\hline
\end{tabular}

Para profundizar en el análisis de estos marcadores de transición, se ha determinado cuál es la relación básica vehiculada por cada tipo de marcador, adoptando para ello la propuesta de Cuenca (2010). Aunque esta autora establece cuatro relaciones semánticas básicas en su clasificación, en este artículo solo se consideran tres de ellas: a) la adición (las oraciones unidas se deben considerar como elementos de una suma de significados), b) el contraste (que implica la presentación de contenidos divergentes respecto del primer enunciado) y c) la consecuencia (que vehicula la relación entre enunciados que presentan una relación de causa-consecuencia). No se toma en consideración la relación que Cuenca (2010) denomina disyunción (formulaciones alternativas de un mismo contenido), ya que estos marcadores siempre introducen contextos definitorios y se tratan de modo independiente en el apartado 3.3.3.

Casi la mitad de los marcadores de transición $(46,7 \%)$ corresponde a la clase marcadores de transición aditivos ${ }^{9}$, seguidos por los marcadores de transición contrastivos $(38,7 \%)$ y los de consecuencia (19,3\%). Los ejemplos (14) y (15) permiten observar el funcionamiento de estos marcadores en contexto; se destaca en negrita el marcador ${ }^{10}$.

(14) Este trabajo presenta una recopilación de fuentes de información que hay sobre que son las lenguas de signos y en especial sobre la lengua de signos catalana (LSC). Muestra también porque Menorca es la única isla de las Islas Baleares que emplea la LSC y no utiliza la LSE como en Mallorca e Ibiza. Además, hablamos sobre la interpretación de la LSC dentro del sistema educativo de Menorca (...). ES_29

${ }^{9}$ Los marcadores aditivos de distribución, que Cuenca sitúa también en el apartado de la adición, son analizados en el apartado 3.3.2. Marcadores estructuradores, siguiendo la clasificación de Hyland (2005) en la que se basa este trabajo.

${ }^{10} \mathrm{Se}$ citan los textos tal y como fueron incluidos en el TFG, independientemente de su calidad lingüística. 
En el ejemplo anterior la estudiante describe su trabajo: presenta las lenguas de signos y la LSC, en particular, expone la particularidad que conduce a que en Menorca se use la LSE y no la LSC para añadir, finalmente, el último tema del trabajo con el marcador de transición aditivo, además.

El ejemplo (15) es una muestra del uso de los marcadores de transición contrastivos, la segunda categoría más presente en el corpus:

(15) Las plataformas lingüísticas normativas dictan una serie de sinónimos con el fin de evitar el uso de amb la que està caient ${ }^{11}$ y sus variantes. No obstante, olvidan el significado pragmático del calco catalán y, en consecuencia, las propuestas normativas no reflejan los significados implícitos típicos de amb la que està caient. ES_07

En este fragmento, la estudiante plantea el ámbito en el que se sitúa su trabajo, el contacto de lenguas en el contexto de Cataluña y su relación con la normativa del catalán. El marcador no obstante permite introducir la motivación del estudio, esto es, las propuestas normativas no consideran aspectos pragmáticos, por lo que la aportación de su TFG será precisamente el análisis pragmático de un corpus de ocurrencias del calco amb la que està caient.

Cabe señalar que los marcadores de transición contrastivos aparecen en el corpus manifestando relaciones de oposición absolutas (como en el ejemplo anterior) o parciales (a pesar de, por poner solo un ejemplo).

Finalmente, mucho menos presentes, los marcadores de consecuencia que aparecen en el corpus son los siguientes: asi pues, consecuentemente, en consecuencia, por consiguiente, por ende. En el ejemplo (16), tras presentar el objetivo, la estudiante introduce la metodología como un efecto lógico de este y así concluye su razonamiento:

(16) El objetivo del presente trabajo es analizar el impacto de la diglosia en la enseñanza del árabe, haciendo hincapié en la dicotomía presente en la EALE (árabe estándar vs. árabe dialectal). Así pues, se ha hecho un estudio cualitativo mediante dos técnicas (...). ES_09

En cuanto a la distribución de los marcadores de transición en los distintos movimientos retóricos identificados (véase Gráfico 2), se observa que la mayoría aparece en el tema y en los resultados. Dada la extensión considerable del primer movimiento, el uso de los marcadores de transición sirve a los autores para cohe-

${ }^{11}$ Esta expresión del catalán es un calco incorrecto de la española con la que está cayendo, expresión metafórica frecuente que se usa para hablar de la inoportunidad de un determinado hecho en medio de una situación especialmente mala o conflictiva. 
sionar su texto, y facilitar el paso del planteamiento del tema a la motivación que sustenta su estudio. Las relaciones semánticas que vehiculan son de adición (2 ocurrencias), consecuencia (6 formas) y, sobre todo, de contraste (9 formas, que suponen un $53 \%$ del total), como en el ejemplo siguiente:

(17) Los resultados de este estudio descriptivo han demostrado que las TIC tienen un impacto positivo en el proceso de enseñanza y aprendizaje del inglés como lengua extranjera, pero a pesar de su evolución, no se han producido diferencias significativas en cuanto los usos reales de las nuevas tecnologías en las aulas. ES_18

Resulta particularmente interesante la tendencia que se observa en los estudiantes de minimizar o limitar el alcance de sus hallazgos con lo que atentan contra la función de promoción que caracteriza a los abstracts; acentúan, de nuevo, su identidad de estudiantes más que la de expertos.

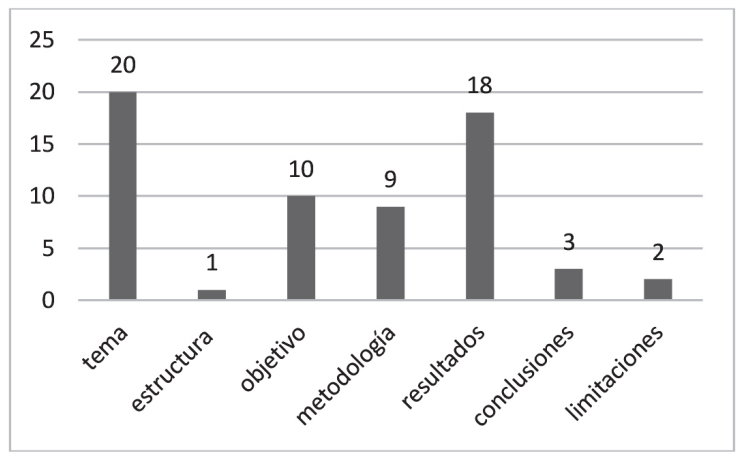

Gráfico 2. Distribución marcadores de transición en función del movimiento retórico.

\subsubsection{Marcadores discursivos estructuradores}

\section{- Marcadores discursivos estructuradores de orden}

Dentro de la categoría de los marcadores discursivos estructuradores se sitúan los marcadores discursivos ordenadores llamados frame markers por Hyland (2005), que coinciden con los marcadores discursivos ordenadores de la información de Martín Zorraquino y Portolés (1999): 
Los ordenadores son estructuradores de la información con dos funciones primordiales: en primer lugar, indican el lugar que ocupa un miembro del discurso en el conjunto de una secuencia discursiva ordenada por partes; $y$, en segundo lugar, presentan el conjunto de esta secuencia como un único comentario y cada parte como un subcomentario (Martín Zorraquino y Portolés, 1999: 4083).

En el corpus se han encontrado un total de 27 marcadores discursivos estructuradores, cuya frecuencia de aparición se representa en la Tabla II. Asimismo, se han identificado diversos hápax: a continuación, en conclusión, en última instancia, por otra parte.

Tabla II. Frecuencia de aparición de los marcadores estructuradores.

\begin{tabular}{|l|l|l|l|}
\hline después & 3 & por otro lado & 3 \\
\hline en primer lugar & 3 & por último & 3 \\
\hline en segundo lugar & 2 & por un lado & 2 \\
\hline finalmente & 7 & & \\
\hline
\end{tabular}

Cabe señalar que estas formas se han localizado en solo 16 de los 36 textos y que su distribución es dispar: 9 abstracts incluyen un único marcador mientras que 18 de las formas se concentran en solo 7 textos, lo que supone un número considerable para este género. Muestra de esta tendencia es el abstract que se incluye a continuación como ejemplo (18):

(18) El objetivo de este trabajo es la creación de un programa de análisis de sentimiento basado en lexicones para tweets en español que sirva de base para futuras experimentaciones y mejoras. En primer lugar, presentaremos y compararemos los enfoques más frecuentemente utilizados para el análisis de sentimiento. A continuación, investigaremos los recursos disponibles actualmente para el análisis de sentimiento en español. Después, explicaremos la estructura y el funcionamiento del programa de análisis de sentimiento creado. Por último, mencionaremos posibles líneas futuras de investigación. ES_35

En cuanto a la distribución de los marcadores estructuradores en función del movimiento retórico, se constata que su frecuencia es mayor en la presentación de los resultados $(30,5 \%)$, donde la función del marcador es organizar los hallazgos o introducir el último enunciado, independientemente del hecho de que haya o no una conclusión. 
Destacan las 6 ocurrencias en el movimiento emergente denominado estructura: habida cuenta que la existencia del movimiento se debe a la confusión del abstract con la introducción al trabajo, al anunciar el contenido del TFG en el abstract, utilizan marcadores estructuradores para presentar las distintas etapas, como en (18).

\section{- Los encapsuladores como marcadores estructuradores}

El uso de encapsuladores es particularmente útil en la redacción de abstracts, unos textos tan breves en los que la presencia de otros marcadores discursivos estructuradores sería poco pertinente. En el corpus, los nombres encapsuladores se usan básicamente para marcar los distintos procesos o fases de la investigación realizada y pueden establecer relaciones tanto anafóricas como catafóricas. El ejemplo (19) es una muestra de encapsulador anafórico y el (20) de un encapsulador que establece una relación de referencia catafórica en el párrafo en el que aparece:

(19) Los resultados muestran que, en cuanto a la forma, las AC más frecuentes son préstamos del castellano (69\% de las AC) y del inglés (26\%). ES_12

(20) Se ha demostrado que el vocabulario es uno de los aspectos más complicados y también más cruciales en el aprendizaje de una lengua extranjera. A pesar de su importancia, los expertos no han empezado a investigar este ámbito hasta las últimas décadas. ES_02

Se han identificado 87 encapsuladores en el texto cuya distribución se presenta en la Tabla III. Además de las formas recogidas en la tabla, se han identificado los siguientes hápax: ámbito, base, características, conclusiones, datos, dificultad, enfoque, evaluación, foco, función, informe, muestra, necesidad, novedades, objeto, obstáculos, problema, propósito, propuesta, pruebas, solución, técnicas, tema, limitaciones, métodos, panorama, programa. Una de las características del corpus es, pues, una gran variedad de formas léxicas encapsuladoras.

Tabla III. Frecuencia de aparición de nombres encapsuladores.

\begin{tabular}{|l|l|l|l|}
\hline análisis & 4 & hipótesis & 3 \\
\hline aspecto & 4 & metodología & 5 \\
\hline elemento & 3 & objetivo & 13 \\
\hline estudio & 3 & pregunta & 2 \\
\hline factor & 3 & propuesta & 2 \\
\hline hecho & 2 & resultado & 19 \\
\hline
\end{tabular}


Como se observa, los encapsuladores más frecuentes son resultado (cf. supra) y objetivo. De hecho, si se observa la distribución de estos encapsuladores en los distintos movimientos retóricos (véase Gráfico 4), se ve que precisamente son más frecuentes en el planteamiento de los objetivos y en la presentación de los resultados; aunque otros procedimientos lingüísticos puedan abrir los movimientos, el uso de los encapsuladores objetivo y resultado ponen de manifiesto la importancia de estos elementos en la construcción discursiva de los abstracts de TFG.

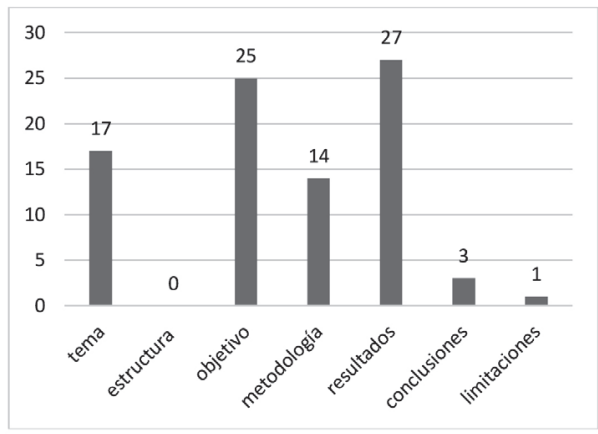

Gráfico 4. Distribución de los encapsuladores en función del movimiento retórico.

\subsubsection{Marcadores de contextos definitorios}

Aunque Hyland (2005) utiliza el término code gloses para referirse exclusivamente a marcadores discursivos reformulativos, en este artículo, siguiendo la línea de otros trabajos anteriores (Alarcón, Bach y Sierra 2007 y Alarcón, Sierra, Aguilar y Bach 2008), se analizan en los textos los contextos definitorios en los que aparecen. Así, se han marcado aquellos fragmentos que, de un modo u otro, explican un término que aparece en el abstract, incluyendo el término reformulado, el marcador discursivo de reformulación y la nueva formulación propuesta. De todos modos, en los ejemplos que se presentan aparece en negrita el recurso reformulativo utilizado.

En realidad, se trata de otro elemento metadiscursivo interactivo, puesto que corresponde a una ayuda que el autor ofrece al lector, basándose en la evaluación que hace sobre el conocimiento del que este dispone. Estas ayudas pueden ser reformulaciones, explicaciones, definiciones o clarificaciones.

En el corpus se han identificado un total de 31 ocurrencias de este procedimiento metadiscursivo. La mayoría $(54,8 \%)$ adoptan la forma de una reformulación mediante el uso de paréntesis, rayas o comas. En estos casos se especifica el término que precede (funciones, fuentes, bloques, etc.), se caracteriza (un total de 26 preguntas (11 cerradas y 15 abiertas)) o se define, como en el ejemplo siguiente 
donde la autora infiere que es probable que muchos lectores no conozcan el referente del término Procés:

(21) En los últimos meses se han vivido en Cataluña los acontecimientos más relevantes del llamado Procés -el conflicto entre Cataluña y el estado español en relación con la independencia de este territorio. ES_23

Merece una atención especial el uso de las siglas, bastante frecuentes en los contextos definitorios (19,3\% del total). En todos los casos, el término precede a la definición: Escola Oficial d'ldiomes (EOI), Televisió d'Eivissa i Formentera (TEF), alternancia de código $(A C)$, etc. La segunda de las formas encontradas en el corpus corresponde a las reformulaciones mediante los marcadores es decir o esto es. Las funciones son diversas: definir el término (las lenguas ficticias, es decir, aquellas pensadas para una situación irreal. ES_32) o manifestar identidad (el estilo de aprendizaje favorito, esto es, la conversación. ES_02). Por último, el uso de los dos puntos para conectar el término con su glosa permite introducir una enumeración de entre dos y cuatro elementos, que el término presenta de forma sintética:

(22) (...) cuatro fuentes distintas: observaciones de campo, entrevistas cualitativas, grupo de discusión y análisis de materiales didácticos; coinciden en tres de sus funciones: participación, interacción y trabajo personal. ES_14

En cuanto a la frecuencia de aparición de los contextos definitorios con los movimientos retóricos de los textos (véase Gráfico 5), se observa que abundan en el tema $(41,4 \%)$ y en la metodología $(44,8 \%)$, siendo ocasionales en los demás. En el tema, el autor se asegura que el lector comprenda los conceptos o la terminología empleados; en la metodología, se detallan los instrumentos empleados o las características de los informantes.

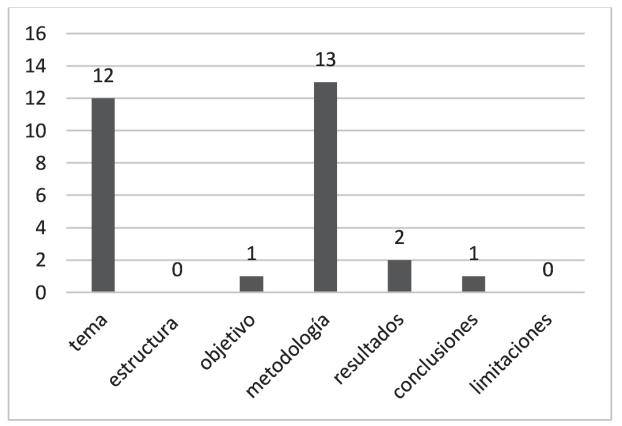

Gráfico 5. Distribución de los contextos definitorios en función del movimiento retórico. 
Por último, cabe señalar que todas las ocurrencias se concentran en 15 de los abstracts: 8 textos presentan una sola ocurrencia mientras que en 7 textos encontramos 21 contextos definitorios. De algún modo, parece que este procedimiento es más característico de algunos autores que de otros. El ejemplo siguiente permite ver cómo la estudiante explicita las dos cadenas de televisión baleares, incluye las siglas correspondientes y reformula el propósito de su trabajo.

(23) en este trabajo se hace un análisis exhaustivo de las políticas lingüísticas del Gobierno Balear y otros organismos, dirigidos a los medios de comunicación de las Islas Baleares. Se tienen en cuenta dos cadenas de televisión, la Televisió Pública de les llles Balears (IB3) i la Televisió d'Eivissa i Formentera (TEF). Se analiza la programación de las emisoras, la lengua que usan y su adecuación, es decir, si en el caso del catalán este se estandardiza o, por el contrario, se usan los dialectos propios de las Islas Baleares. ES_19

Para terminar este apartado del estudio, es preciso subrayar la importancia de los elementos estructuradores en la redacción de los abstracts por parte de los estudiantes. En efecto, como se puede observar en el Gráfico 6, más de la mitad de los marcadores discursivos utilizados en los textos corresponden a la categoría estructuradores, que contiene tanto los encapsuladores (42\%) como los marcadores estructuradores de orden (13\%).
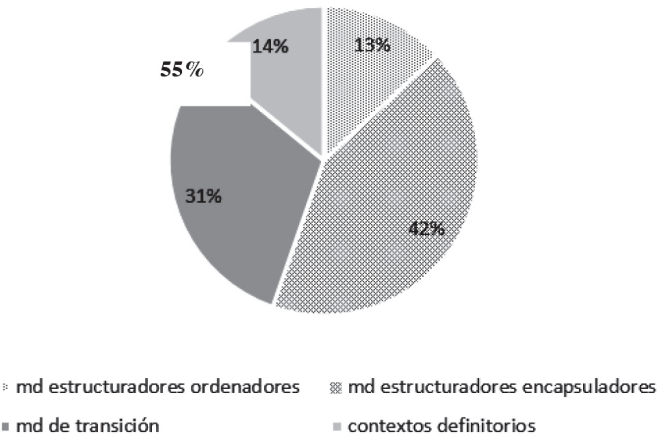

Gráfico 6. Porcentajes de uso de las distintas categorías de marcadores.

\section{CONCLUSIONES}

Del análisis realizado se desprende que los abstracts redactados por futuros egresados son textos híbridos, a medio camino entre el discurso académico, producido por un estudiante que va a ser evaluado y un discurso especializado, producido 
por y dirigido a lectores expertos. Los problemas detectados en la redacción de estos textos permiten concluir que, para los estudiantes, el género abstract de TFG es un género oculto, según la terminología de Swales (1996), es decir, un género textual cuyas características no son, por uno u otro motivo, conocidas por aquellos que deben producirlos. Así, la hibridez a la que nos referimos se manifiesta en una identidad doble del autor, con rasgos propios de su situación de estudiante y otros propios de un investigador principiante.

La identidad del aprendiente se observa en distintos planos: el supratextual (mención de la lengua español en el título), el macrotextual (la presencia de los movimientos emergentes detectados -estructura y limitaciones) y, también, a nivel microtextual en tres aspectos concretos: a) el uso de tiempos verbales en futuro, que no da la imagen de investigación terminada cuyos resultados se quieren compartir con el lector, sino de trabajo por empezar; b) el elevado uso de marcadores de transición con significado contrastivo en el movimiento resultados, introduciendo limitaciones al alcance de los mismos; c) el uso de contextos definitorios, básicamente en los movimientos tema y metodología, para mostrar a los evaluadores del TFG que se poseen los conocimientos necesarios en la disciplina en la que este se sitúa.

En cuanto a la segunda identidad, esto es, la de investigadores principiantes, se manifiesta gracias a la presencia destacada de los movimientos clásicos de los abstracts (tema, objetivo, metodología, resultados; y en menor grado, conclusiones). También destaca el uso de marcadores discursivos estructuradores, básicamente de encapsuladores, para establecer la cohesión textual: como se ha mencionado, se trata de un rasgo típico de los abstracts producidos por investigadores expertos.

Ser competente discursivamente significa haber interiorizado las características de un género determinado a nivel no solo lingüístico, sino también a nivel sociocultural y a nivel pragmático, es decir, en relación directa con el contexto en el que el género se produce y se recibe. Esta investigación pone de manifiesto algunas carencias en cuanto al grado de dominio de esta competencia por parte de los estudiantes. Las implicaciones de este trabajo son claras: como investigadores y formadores de futuros egresados, es de vital importancia que se faciliten a los estudiantes aquellos conocimientos que permitan empoderarlos en cuanto al uso del género abstract y al dominio de los géneros discursivos. Es una necesidad no solo para aquellos que decidan iniciar estudios de máster y de doctorado, sino también para aquellos que vayan a integrarse en el ámbito profesional. Así pues, desde el punto de vista de la didáctica de la escritura, ayudar a los estudiantes a alcanzar la competencia discursiva supone hacerles conscientes de las características del género a nivel supratextual, macrotextual y microtextual. Esta toma de conciencia pasa obviamente por la descripción minuciosa del género, una descripción que debería estar directamente relacionada con la evaluación que se haga de los textos escritos por los estudiantes, puesto que cada uno de los rasgos definitorios del género debería ser un indicador de calidad de las producciones. 


\section{REFERENCIAS}

Adelstein, Andreína y Kuguel, Inés. (2005). Los textos académicos en el nivel universitario. Buenos Aires, Argentina: Universidad Nacional de General Sarmiento. Aktas, Rahime Nur y Cortes, Viviana. (2008). Shell nouns as cohesive devices in published and ESL student writing. Journal of English for Academic Purposes, 7, 3-14.

Alarcón, Rodrigo; Bach, Carme y Sierra, Gerardo. (2007). Extracción de contextos definitorios hacia la elaboración de corpus especializados: de una herramienta de ayuda terminológica. Revista Española de Lingüística 37, 247-278.

Alarcón, Rodrigo; Sierra, Gerardo, Aguilar, César y Bach, Carme. (2008). Definitional verbal patterns for semantic relation extraction. Terminology, 14(1), 74-98.

Bathia, Vijay K. (1993). Analysing Genre - Language Use in Professional Settings, Londres, Reino Unido: Longman.

Borreguero, Margarita. (2018). Los encapsuladores anafóricos: una propuesta de clasificación. Caplletra, 64, 179-203.

Cassany, Daniel y López-Ferrero, Carmen. (coord.) (2000). Centro de redacción de la UPF. Disponible en https://parles.upf.edu/llocs/cr/casacd/index.html [Consulta: 12/12/2019].

Cavalieri, Silvia. (2014). Variation Across Disciplines. The case of Applied Linguistics and Medicine. En: Bondi, Marina y Lorés, Rosa. Abstracts in Academic Discourse: Variation and Change. (161-174). Berna, Suiza: Peter Lang.

Cuenca, Maria Josep. (2010). Grámatica del texto. Madrid, España: Arco/Libros.

Da Cunha, Iria. (2016). El trabajo de fin de grado y de máster. Redacción, defensa y publicación. Barcelona, España: UOC.

Díez Prados, Mercedes. (2018). Abstracts nouns as metadiscursive shells in academic discourse. Caplletra, 64, 153-178.

Dos Santos, Mauro Bittencourt. (1996). The textual organization of research paper abstracts in applied linguistics, Text, 16(4), 481-499.

Francis, Gill. (1986). Anaphoric nouns. Discourse Analysis Monographs II. Birmingham, Reino Unido: University of Birmingham.

Graetz, Naomi. (1985). Teaching EFL students to extract structural information from abstracts. En: Uljin, Jan M. y Pugh, Anthony K. (eds.). Reading for professional purposes. (123-135) Leuven, Bélgica: ACCCO.

Halliday, Michael y Hasan, Ruqaiya. (1976). Cohesion in English. Londres, Reino Unido: Longman.

Hyland, Ken. (2005). Metadiscourse. Londres, Reino Unido: Continuum.

Jiang, Feng y Hyland, Ken. (2017). Metadiscursive nouns: Interaction and cohesion in abstract moves. English for Specific Purposes, 46, 1-14. 
Jiang, Feng y Hyland, Ken. (2018). Nouns and academic interactions: A neglected feature of metadiscourse. Applied Linguistics, 39(4), 508-531.

Lorés Sanz, Rosa y Bondi, Marina. (2014). Introduction. En: Lorés Sanz, Rosa y Bondi, Marina. Abstracts in Academic Discourse. Variation and Change. (9-17) Berna, Suiza: Peter Lang.

López Samaniego, Anna. (2018). La encapsulación nominal en el discurso académico-científico oral y escrito: patrones de aparición. Caplletra, 64, 227-252.

Martín Peris, Ernesto (dir.); Atienza, Encarna; González, Vicenta; López-Ferrero, Carmen y Torner, Sergi. (2008). Diccionario de términos clave de ELE. Madrid, España: SGEL. Disponible en: https://crc.cervantes.es/Ensenanza/biblioteca_ ele/diccio_ele/default.htm [Consulta: 11/11/2019].

Martín Zorraquino, M. Antonia y Portolés, José. (1999). Los marcadores del discurso. En: Bosque, Ignacio y Demonte, Violeta (dir.) Gramática descriptiva de la lengua española, 3. (4051-4213) Madrid, España: Espasa.

Muñoz, Verónica L. y Ciapuscio, Guiomar E. (2019). Los nombres rotuladores: un estudio de los rótulos cohesivos en artículos de investigación en inglés y español. Revista Signos. Estudios de lingüística, 52(100), 688-714.

Parodi, Giovanni. (2010a). La organización retórica del género. Manual a través de cuatro disciplinas: ¿cómo se comunica y difunde la ciencia en diferentes contextos universitarios? Revista Boletín de lingüística 22(33), 43-69.

Parodi, G. (2010b). The rhetorical organization of the Textbook across disciplines: A 'colony-in-loops'? Discourse Studies, 12(2), 195-222.

Parodi, Giovanni y Burdiles, Gina. (2016). Encapsulación y tipos de coherencia referencial y relacional: el pronombre ello como mecanismo encapsulador en el discurso escrito de la economía. Onomázein, 33, 107-129.

Saeeaw, Supachai y Tangkiengsirisin, Supong. (2014). Rethorical Variation across Research Article Abstracts in Environmental Science and Applied Linguistics. English Language Teaching, 7(8), 81-93.

Sancho, Jordi. (2014). Com escriure i presentar el millor treball acadèmic: guia pràctica per a estudiants i professors. Vic, España: Eumo.

Schmid, Hans-Jörg. (2000). English abstract nouns as conceptual shells: from corpus to cognition. Berlín, Alemania: Mouton de Gruyter.

Schmid, Hans-Jörg. (2018). Shell nouns in English. A personal roundup. Caplletra, 64, 109-128.

Swales, John. (1990). Genre Analysis: English in Academic and Research Settings. Cambridge, Reino Unido: Cambridge University Press.

Swales, John. (1996). Occluded genres in the academy: The case of the submission letter. En: Ventola Eija y Mauranen Anna (eds). Academia Writing: Intercultural and Textual Issues. Amsterdam, Holanda: John Benjamins, 45-58. 
Venegas, René, Núñez, M. Teresa, Zamora, Sofía y Santana, Andrea. (2015). Escribir desde la pedagogía del género. Guías para Escribir el Trabajo Final de Grado en Licenciatura. Valparaíso, Chile: Ediciones Universitarias de Valparaíso, Pontificia Universidad Católica de Valparaíso.

Venegas, René, Zamora, Sofía y Galdames, Amparo. (2016). Hacia un modelo retórico-discursivo del macrogénero trabajo final de grado en Licenciatura. Signos, 49(1), 247-279.

Vendler, Zeno. (1968). Adjectives and nominalizations. París, Francia: The Hague.

\section{(FOOTNOTES)}

1. The labels in the examples coincide with those of the WOCAE corpus. CH-ES correspond to the name and language of the corpus, followed by the academic year and the example (_4_36) and, after the two dots, the line of the transcript in which the example is included. However, since the loading of the corpus is still in progress, some example may be missing, but will be visible soon.

2. The English translation of the Spanish/FL examples does not contain the lexical and grammatical errors of the original student production. 